\title{
Research on New Network Architecture Through Sd-Wan
}

\author{
Sallauddin Mohmmad, Dadi Ramesh, Syed Nawaz Pasha, Shabana, Kotha Shankar
}

\begin{abstract}
Todaysocietyandmanyorganizationsusetheinternetwhi chisinterconnectsthepeoplearoundtheworld.Thisinternetusesthetr aditionalWideAreaNetworkarchitecture.ThetraditionalWANrequi resasingleprovidertoaccessmanysiteswhichdoesallowthesitesfordir ectaccesstocloudresources, inflexibleandthesearehadunpredictabl eperformance.ToovercometheseproblemsSD-

WANarchitectureintroducedbasedonSDNtechnology.SD-

WANhaddynamicmulti-

pathselectionandcloudoptimizedfunctionalities.Thesefunctionaliti esareprovidingimprovedperformanceandfaulttolerance.Theimple mentationofSD-

WANininterautonomoussystemswithCloudComputingallowsvirtu alizationonthenetworkarchitecturewithprovisioninganddeprovisioningofresourcesondemand,soitisbecomingneweraofservic ebyCloudComputingnamedasNetworkasaService(NaaS).Recently thetelecomservicesstartedtheNaaStotheirsubscriberstoavoidthemo reuseofphysicalnetwork.Duetopresentnetworktrafficdemands $S D$ WANneedsdynamicnetworkcontrols, optimalpathselections, Forwa rderrorcontrolsandsecuretransmission.Inthisarticlewediscussabo utSD-
\end{abstract}

WANdesignissues,transmissioncontrolandpossibleintegratedarchi tecture.wealsopresentthefunctionalprocessofRESTfulAPIonSDWANwithprogramfragments.

KEYWORDSSD-WAN,dynamicmultipathselection,NetworkasaService,RESTfulAPI

\section{INTRODUCTION}

Intoday'sworldtheusageoftheinternetiseverywhere.Theusa geofthebasicprotocolsismoreandneednewadvancedprotocolst oimprovetheefficiencyoftheinternet.Todayinternetnotonlyus edfortheacademiccommunitybutitsusabilityisexpandtoconsu merorientedusecases.Inordertodeploynewadvancedprotocols needstandardizationthroughInternetEngineeringTaskForce(I ETF).Thatprocesswasslow.Asaresultofthisresearchersarestro nglyrecommendedtodevelopprogrammablenetworktoacceler atetheusageofinternetovertheWorldWideWeb.

Inthiscontext,firstefforttoachieveprogrammablenetworkw asActiveNetworkArchitecture[1].InANAworkingprocedurel oadsthecustomizeddesignprogramsintonetworkdevices.Thes eprogramsperformcorrespondingfunctionsinthenodeatthetim eofwhenapackettraversedthroughit.Theseprogramsareembed dedanduploadedinthenetworkdevicebythethirdparty.Thirdpar

Revised Manuscript Received on April 12, 2019.

SallauddinMohmmad,Department of Computer Science \&Engineering,S R Engineering College,Warangal, TelanaganaState,India.

Dadi Ramesh,Department of Computer Science \&Engineering,S R Engineering College,Warangal, TelanaganaState,India.

Syed Nawaz Pasha,Department of Computer Science \&Engineering,S R Engineering College,Warangal, TelanaganaState, India.

Shabana,Department of Computer Science \&Engineering,Sumathi Reddy Institute of Technology for Women ,Warangal, TelanaganaState,India.

Kotha Shankar,Systems Engineer,Tata Consultancy Services(TCS),HYD,Telangana, India. tiesaredevelopinganddeploycustomfunctionalityfortherespec tivenetworkdevice. TheActiveNetworkscreatenewnetworktre ndandtechnologiesinthenetworkarchitecture.Thesearefullyne tworkprogrammed.TheActiveNetworksarebrokemanyconve ntionalprimitives. Thesearewidelyusedanddeployedinthenetw orks. TheActiveNetworksareleadustotheSoftwareDefinedNet work(SDN)[1][2].

Theprogrammablenetworkneedscontroloverthenetworking protocolstogaincontrollability.Soweneedtoseparatethecontrol anddataplaneinthenetworkarchitecture.Bythiswecangetmore generalizedcontrolprotocols.OpenFLowwasthefirstprominen tefforttoaprogrammablenetworkingarchitecturemeansSoftwa reDefinedNetwork(SDN)[2].OpenFlowmainlyfocusedonthet womajorinsights.First,itaimstoanetworkwithgeneralizednetw orkdevicesandfunctions.Secondlyittellsaboutnotationswithre specttoNetworkOperatingSystem.

OpenFlowconsistingofasoftwarewhichisasimplifiedAPIan dprogrammingabstractionstodefinedapplicationstocontrolan dmanagethenetwork. TheAPIsupportsanylanguagestowritene tworkpolicestocontrolthenetworkoperatingsystem. Theseprog ramsarestandardizedthenetworktogaincontrollability.OpenFl owisnottheonlyprotocolthatcanbeimplementingtheSDNarchi tecture[3].PathComputationElement(PCE)architectureisused forcentralizedcontroltrafficInthenetworktopology.ThePathC omputationElementProtocol(PCEP)isalsousedforsamepurpo seinthenetworktopology.

SoftwareDefinedNetworks(SDN)aresimplifiedthewholene tworkarchitecturebysimplifyingfunctionalitiesofnetworkdevi ces. Thesenetworkdevicesareverysimple,automatedanduninte lligentboxesthathavejobofhandlingpacketsbasedtransmission withrespecttotherulesofcontrolplane.SDN-

enabledforwardingelementstoperformactionaccordingtomatc hingpattern,meanswhenanetworkpacketpassingthroughadevi cesuchasforwardordropinthatdeviceneedstobematchingwitht hepacketheadersaspertheruledefinedbythecontrollogic.Basic allySDNarchitecturehasbeendatacentricsettings.

OntheotherhandWideAreaNetworksweremoreexpensiveto thepresentnetworkarchitectureanditalsodifficulttomanagebec ausetheyconsistsofhigh

performancerouterstohandlehugedata,thisleadstoincreasingc apitalexpenditure(CAPEX)aswellascomplexconfigurationre quiringextensivemanagementeffortsincreasingOPEXandfail ureprobability.Tosolvealltheseproblemsresearchersaregiving moreprioritytotheSDNforWideAreaNetwork[4].Thiscanesta blishnewnetworkarchitecturewithcentralizedcontrolandloadp lanningtodistributeloadmorewisely, adapttovarious.

Published By:

Blue Eyes Intelligence Engineering \& Sciences Publication 
Presently,societymorereliesonthenetworktointerconnectwi ththepeople,devicesandcontent.Thenetworksarebecomesdail yroutine.Theinternetinterconnectsallthings.Sothenetworksar eprovidemorevaluetotheuser.Programmablesystemsareprovi dingefficientapplicationstocloudtoserveITutilityforaglobaliz edandmobileworkforce.ImprovementofMulti-

ProtocolLabelSwitching(MPLS)andCarrierEthernet(CE)had provideservicestotheWAN.Inthisinterconnectionofnewsites wereanissue, servicechanges, bandwidthchangesandcloudserv iceswerethemajorchallengesfortheWAN.SD-

WANwasemergedasoneofthesolutionforaddresstheseissues. TypicalWANnetworkarchitecturehadcomplexity.Byusingthe SD-WANwecanreducethenetworkcomplexityalot.SD-

WANnetworkarchitecturehadcentralizedcontrolprograms.Th eseprogramsimprovetheapplicationperformance.SD-

WANusesdynamicmultipathselection. Thisimprovestheperfor manceofnetworkandalsoprovidesredundancyforaWAN.Asin glewebbasedportalprovidesrealtimeanalyticsforeveryconnect edsiteintheSDWAN.Bythiseachsitecandiagnosetheperforma nceoftotalnetwork.InthisarticlewediscussaboutSD-

WANdesignissues, transmissioncontrolandpossibleintegrated architecture.wealsopresentthefunctionalprocessofRESTfulA PIonSD-

WANwithprogramfragments.Section2tellaboutthesurveyabo utthetopicincludingtechnicalissues.section3tellaboutrealtime implementationusingRESTfulAPIinterfaceinthenetwork.Sect ion4describethebasicSD-

WANarchitecturewithassociationCloud.Section5discussthef ewchallengesovertheSD-WAN.

\section{RELATEDWORK}

InseparationofnetworkdevicesandcontroloperationstheOp enFlowprotocolplayedtheimportantroleandcreatedtheprogra mmablecontrollingonnetwork[3].OpenFlowinsistedthetwom ajorthinginSDN.First,itcreatedthegeneralizationfunctioningf ornetworkdevicesbyasimplepacketbasedmatch-

actionpattern.Secondly,OpenFlowcreatedthenecessityofNet workOperatingSystem.Inthisnetworkrevolutiondifferentkind ofAPIarecontrollingthenetworkspartswiththesupportofNOS. APIisataskbasedsimplifiedprogrammingabstractionwhichcan handlethespecificnetworkoperationornetworkphysicalpart.Su chkindofAPIcanabletodevelopbyanyprogrammingplatform. Asdiscussedearlier,OpenFlowalsoaapplicationbasedprotocol. OpenFlowcreatethecommunicationbetweenthecontrolanddat aplanes.FromthedataplaneOpenFlowobtainthetopologicalstr ucturesdynamicallywiththesupportofPCEandforwardpacketfl owobtainPCEP.AswellsomeothersupportingprotocolsforOpe nFlowareNETCONF,RESTCONF, andOVSDB.IntheSDNthe majorfunctionalityimplementedasasoftware-

basedvirtualnetworkfunction(VNF)whichmayrunonavirtual CustomerPremisesEquipment(CPE)attheclientpremisesoran yothertypeofgeneralizedcomputeplatform,e.g.,serverinadatac entre, whichmayalsobemanagedbythebyacloudserviceprovide r[4][5].Inpresentdaysthenetworkalsobecomingintovirtualizat ionbecauseofSDNintegratedCloudtechnology.Cloudcomputi ngintroducedthenewserviceasNetworkasaService.

\subsection{NetworkasaService}

CloudComputingsupportingforvirtualizationonthenetwork architecturesandcontrollingsystemsothatitisbecomingneware aofresearchinthepresentdays.thatservicenamedasNetworkasa
Service(NaaS).Withrespecttothatthenewcustomerofcloudcan takerentalnetworkitselfwithoutestablishingthephysicalnetwo rk. Theusercanabletoaccessthenetworkasaservicewhereeverh esit.Itjustreducetheeffortsofserviceholder.Insimilarmannerth evirtualizednetworkcontrolisavailablefromthecontrolplanew hichcanmanagethevirtualizednetworktopology.theimplement ationofsuchnetworkisneedwithOpenFlowbaseddevices.Prese ntlyitisspreadinginallkindofnetworksystemslikedatacentrenet work,mobilecommunicationnetworksandsatellitenetworksetc .EvenIfthistechnologyimplement50\%ofnetworkthenitwillred ucetheuseofnetworkphysicalarchitectures,networkinvestmen ts, datatransmissionproblems, reducetheuseofmorebandwidth, packettracingsandproblemofphysicaldamagingofdata.

RecentlythetelecomservicesstartedtheNaaStotheirsubscrib erstoavoidthemoreuseofphysicalnetworkandtheyintegratedin WANalso.GoogleimplementedtheSDNontheirdatacentrenet works.Toreducetheuseofdumbpipesnowaday'stelecomoperat orsstartedtheNaaSserviceusingcloud.BasicallythisNaaSservi cesareimplementedinthecloudusingWANconnectivitywhichi sbasedonSDN.typicallyNaaSservicesarelikedatacentrenetwo rk,telecominternetservicesandBandwidthonDemand.Datacen tres(DCs)networkprovisioningandde-

provisioningappliedintheglobalnetworksystem.Consequently whichcomesundertheWideAreaSoftwareDefinedNetworking (WA-

SDN).GoogleandAWSareestablishedtheirdatacentresinallcor nersoftheworld.Thevirtualizationofdatanodeusingthecloudca nbedonebyIaaS.Buttobringtheirdatacentresnetworkintothevir tualizationisachallengingfactor.Cloudtechnologyshouldappli edineveryedgeofnetworkwhichmayalsoinWAN.Theimpleme ntationofSDNininterautonomoussystemsiscalledasSoftware DefinedWideAreaNetwork(SD-WAN).

\subsection{ManagedSD-WANService}

SD-

WANconsequentlybecomepopularintheeraSoftwareDefined NetworkwhichisappliedonWANconnection,thistechnologyin tegrationusedtoconnectedtheenterprisenetworksincludingthei rbranchesanddataservers.generallytheWANnetworkreachtos witchbytakinginmanyhopstorach.WANcontainsoneormoreau tonomoussystems.thecommunicationandtransmissionlatency arehigherthanthenormalnetwork. Sothatwiththe singlecontrolsystemwecan'tcontrolnetworkoptimally. Whichl eadstoestablishmulticontrolsysteminSDN.VerypopularSDWANbasednetworkisGoogle'sB4networkwhichisalargestim plementedSDNbasednetworkwithmultiplenumberofSDNcon troller[6].

\subsection{SD-WANtrafficpathcontrol}

SDWANhasbeenbroadlyfollowed,mostlybecauseofSDW ANvendorswhooffernumerousandproprietarypathandnicema nagecapabilities.LeadingSDWANcompanieslikeVelocloud, Viptela,VersaNetworks,Cisco,Juniper,Riverbed,Silverpeaka ndCitrix,serveorganizationcustomersbothbyusingpromoting SDWANanswersdirectlytothemorwiththeaidofpresentingthe equaltotelcosinordertoprovidecontrolledSDservicestoorganis ationcustomers.courseandhigh- 
qualitymanipulatefeaturessuppliedbymeansofeverySD-

WANdealerareslightlyoneofakindfromsuppliertoseller(much likemobileVideoOptimizationcarriershadtheirownoptimizati onmethodssomeyearsinthepast).SD-

WANcontrollerisresponsiblefordeliveringpoliciestoeachSDWANCPEandisavailableinmanydifferentconfigurations[1][6 ].

\subsubsection{PathselectionbyapplicationandDynamicPathControl(D} $P C)$

Thefirststepisselectingthepathsfortheapplicationforexampl ethepathsforMPLSpathforVoIP,videoconferencingandERP, $b$ utInternetforothers.AnMPLScommunity,eventhoughextraor dinarilyhighlypricedcomparedtobroadbandinternet,doesprovi dehighgreatcarrier.however,broadbandnetservicedoesnotincl udeSLAlikeMPSVPNdoes,sothere'snoassureregardinglatenc yorpacketloss.So,yougetwhatyouget,anditoftenforhomeusers

Basic: Path selection by application

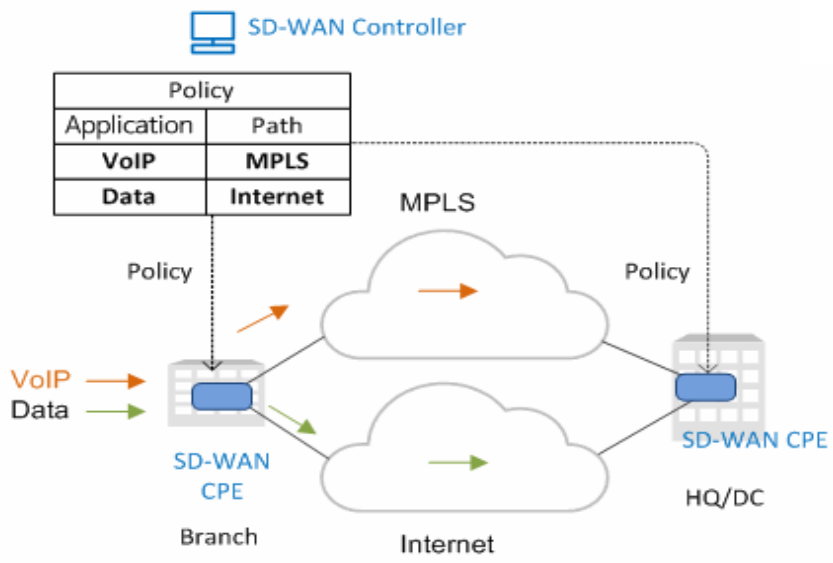

.forthisreason,therewerereasonableconcernsamongstorganiza tionsapproximatelyhandingoverespeciallyessentialutilitytraff iclikeVoIP,videoconferencing,ERP,andsoon.MPLSandintern etarepacketnetworks.Packetnetworksaregenerallyatthedange rofgoingthroughcongestion.therefore, whatSDWANanswerw antmostisacoursecontrolsolutionthatallowsforeverysoftwaret odynamicallytransfertheirpathsinactualtime, inresponsetoco mmunitysituations,asopposedtostickingtoatleastonespecificn etwork.DPCensureseachSD-

WANCPEmonitorsthequalityofmultipletunnels(i.e.,latency,p acketloss,jitter,throughput)inrealtime.If'low-

loss"policyisappliedandDPCisenabled,aVoIPapplicationguar anteesthatVoIPpacketsaredeliveredthroughthebestpossiblepa thatalltimebydynamicallyswitchingbetweenMPLSandInterne tpathsdependingonthepacketlossconditionsinthenetwork(Not e:Itisdifferentfromloadbalancingbetweentwopaths).

\section{Dynamic Path Control (DPC)}

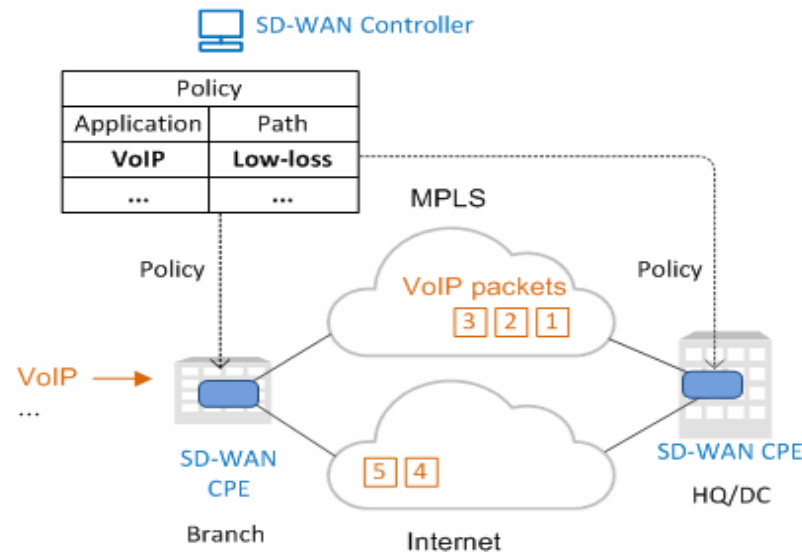

Figure 1. Path selection and Dynamic Path Control in SD-WAN

\subsubsection{TunnelBondingandFEC(ForwardErrorControl)}

ForapplicationssuchasDataRelation,Backup,andFileTransf er,theperformanceoftheseapplicationsdependsontheavailable bandwidth.Timeisoptimalwhenonepathisutilizedratherthanall availablepaths"TunnelBonding"performsper-

packetloadbalancinganddistributethepacketsbelongingtoonef lowtodifferentWANpathsandhenceachievingmaximumthrou ghput.ThebasicconceptofthetechnologyisquitesimilartoMulti

PathTCP(MPTCP), thathasbeenadoptedincommercialnetwor ksinSouthKorea.Thedrawbackofthissolutionisthatthepackets
arenotintheoriginalorderassentbythesender.Toaddressthisissu e,SilverPeakintroducedPacketOrderCorrection(POC), aresequencingprocessperformedbySD-

WANCPEonthereceivingend.InInternetnetworks,packetlossi snotguaranteed.Sopacketssomehowlostonthewaywhichtrigge rTCPretransmissionandsignificantlylowersTCPthroughput.A sasolutiontothisSilverPeakcameupwithwastoinsertFECpacke tstosomewhereamongthepacketsbeingtransmitted[7].Asseeni nthefigurebelow,theFECpacketmaybesentthroughMPLSsepa rately,orthroughtheInternetwithotherpackets. 

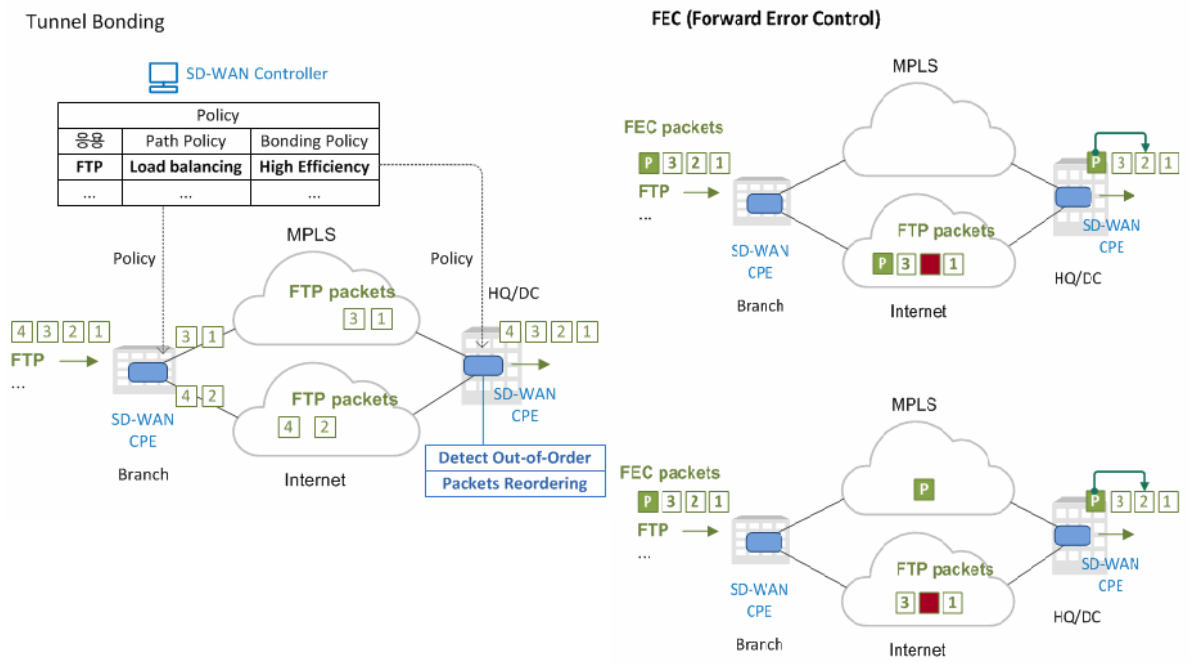

Figure2.TunnelBondingandForwardErrorControlinSD-WAN

\section{APPLICATIONINTERFACE}

InSDN, thedataplaneandthecontrolplanecaninteractwithina closedcontrolloopInitiallythecontrolplanereceiveseventsfrom dataplaneandthenitcomputessomenetworkoperationsbasedon theevents.Thedataplanethenexecutestherequiredoperationsw hichcanchangethenetworkstates. Anetworkisacomplexstructu rewhosefunction,resourceandbehaviourvariesdynamically. T heNBIofthenetworkhastoadoptaccordingtotheevolvingnetwo rkstructureandtheapplicationparadigmshifts.AsaresultRESTf ulNBIhasbeendesignedtomanagedistributedresourcessuchas datanetworking.ThebenefitofRESTAPIisitprovidesdifferentf unctionsatthesametime.ARESTAPIiscapableofachievingsuc hflexibilitythroughthemechanismofhypertext-

drivennavigationofconnectedresources, whichisalsodescribed as"hypertextastheengineofapplicationstate"in[8].TheSDNco ntrollercomeswithalogically-

centralizednetworkabstractionforapplicationstomanagethene tworkinanefficientandflexiblemanner.Figure2illustratesthein ternalarchitectureofatypicalSDNcontrollerbasedonthemodel ofOpenFlow.AtthebottomistheOpenFlow-
basednetworkabstraction.Thecontrollermaintainsaglobaltopo logyofthenetworkwhichwillconfiguretheAPItomanagetheflo wtablesintheunderlyingOpenFlowdevices.Basedonthisconfig urationAPI,built-inapplicationscanbeabletoimplementhighlevelnetworkabstractions, suchasvirtualnetworks, thatprovide anefficientwaytomanagemoderndatanetworks, especiallyfort helargescaledatanetworksindatacentresandcloudcomputingpl atforms.

TheOpenFlowconfigurationAPIrequiresapplicationstodevi seflowentriesacrossvariousOpenFlowdevices(e.g.switches/ro uters).Someofthedeclarativeconfigurationlanguages, suchasF low-

basedManagementLanguage(FML),Procera,andFrenetic,are proposedtoexposenetworkprogrammabilitybyexpressingthes emanticsofnetworkpoliciesdeclaratively,andthepolicylayer,w hichisgenerallybuiltontopofexistingcontrollers, suchasNOX, willcompilethehigh-

levelpoliciesintotheflowconstraintstobeenforcedbythecontrol ler.AlthoughtheseAPIsareeffectiveinsomeapplications,theyar enotasreliableasRESTfulservices.

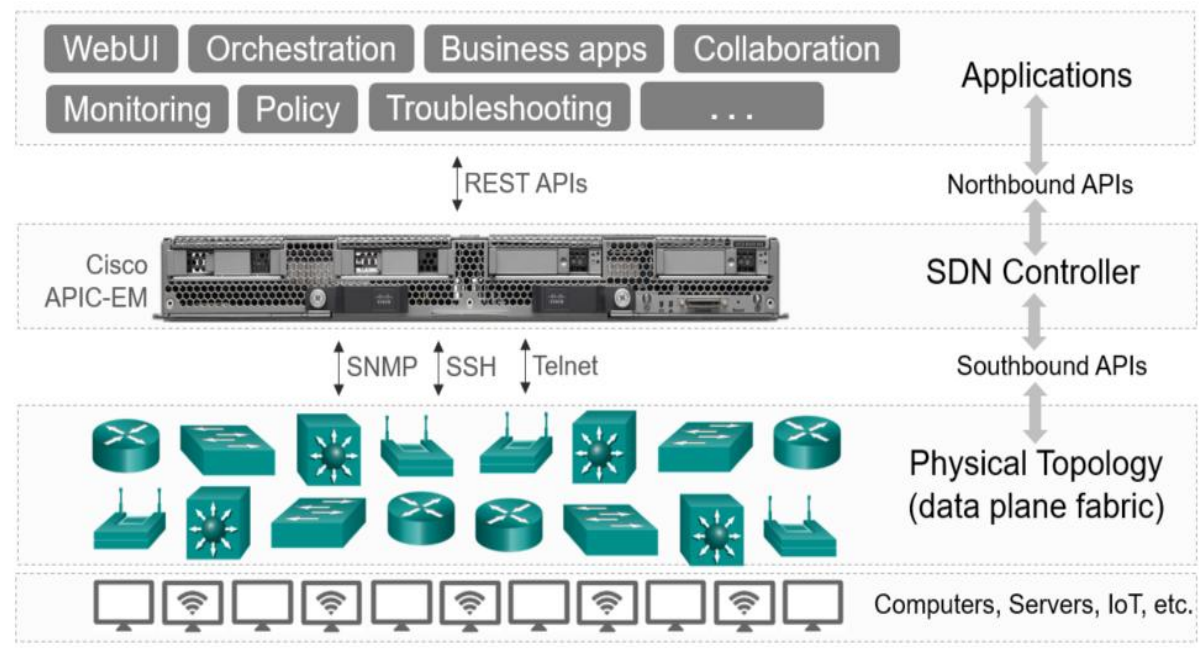

Figure 3. SDN based application interface for SD-WAN 
TheRESTinterfacemakesuseofHTTPGET,PUT,POST, and DELETEoperationsagainstadefinedsetofURLsrepresentingG Oresources. ThetransfertaskrequiresausertoissuesaPOST tohtt ps://transfer.api.globusonline.org/v0.10/transferwithadocum entdescribingthetransferrequest,including,forexample,source anddestinationendpointsandfilepathsandoptionsandtoaccesst hestatusofatask,theuserissuesaGETrequesttohttps://transfer.a pi.globusonline.org/v0.10/task/<task_id >;thesystemthenretu rnsadocumentwiththestatusinformation.TheRESTinterfaceis versioned,soGOcanevolveitsRESTinterfacewithoutbreaking existingclients.DocumentsthatarepassedtoandfromHTTPreq uestsareformattedusingJavaScriptObjectNotation(JSON)and ExtensibleMark-

upLanguage(XML).SupportedsecuritymechanismsincludeH TTPSmutualauthenticationwithanX.509clientcertificateand(f orWebbrowsers)HTTPSserverauthenticationwithcookiebasedclientauthentication. TheWebinterfaceareincorporatedo ntheRESTinterfaceusingstandardAsynchronousJavaScript(A JAX)andXMLtechniques.AGOWebpagecontainsstandardHT ML,CSS, andJavaScript,interactingwiththeRESTinterfacethr oughstandard-

sessioncookiebasedclientauthentication.TheWebGUIsupport sbrowsingremotefilesystems, aswellassubmitting,monitoring, andcancellingtransferrequests.

IntheSD-

WANtocommunicatetheclienttoserveroranyproxyserverrequ estandresponsescreatedmostprobablybytheRESTfulAPI.Whi chisaninterfacebetweenApplicationsanSDNcontrollerintheN orthbound.TheRESTfulrequestelementconsistingofmethod, URL, authentication,customerheadersandrequestbodyinthepr ogramfragment.ThemethodsarelikeGET,POST,PUTandDEL ETEandtheAuthenticationprovidedbybasicHTTP,OAuthand CustomfinallytherequestbodycancreatedbyJSONorXML.Aut henticationofaRESTfulrequestisprocessedbyfourways:

None:TheAPIresourceispublicandanybodycanplacethereq uest.

BasicHTTP:Theusernameandpasswordareforwardedtothes erverinanencryptedformat

Token:AsecretkeywhichiscreatedandprovidedfromtheWeb APIdeveloperportal.

OpenAuthorization(OAuth):Anopenstandardforretrievinga naccesstokenfromanIdentityProvider.Thetokenisthenpassed witheachAPIcall.

Foryourpurposes,youwillusetokenauthentication. Toaddato kentoyourRESTfulrequest,simplyaddkey=your_api_key,ass howninbelowfigure.

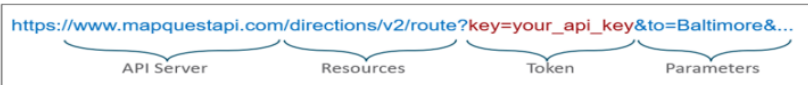

Figure4.RESTfulAPIrequestparametersforrequestaddre ssinSD-WANnetwork.

\subsection{CreateVariablesforAPIRequest}

RESTfulrequestinputURLisacombinationoffollowingvaria bles:

main_api-themainURLthatyouareaccessing

orig-theparametertospecifyyourpointoforigin

dest-theparametertospecifyyourdestination

key-

theMapQuestAPIkeyyouretrievedfromthedeveloperwebsite. main_api="https://www.mapquestapi.com/directions/v2/r oute?"

orig="Washington"

dest="Baltimaore"

key="your_api_key"

Createavariableurltostoretheresponsefromourrequest.

url=main_api+urllib.parse.urlencode(\{ "key:key","from":o rig,"to":dest \})

Aftercreatedtheseprogramfragmentswecanmakearequestb ycreatingthejson_datavariable,Thevariablemakesuseoftheget methodoftherequestsmoduleandspecifiesJSONastherequeste dformat.Theprintstatementisusedtotestthattherequestwassucc essful.

json_data=requests.get(url).json()

print(json_data)

Requestshasbuilts-

inJSON, whichyoucanusetogetresponseinJSONformatagain. importrequests

importjson

req=requests.get('https://xyz.com/timeline.json')

req.json()

Rewritetheoriganddesttobewithinawhileloopinwhichitrequ estsuserinputforthestartinglocationanddestination.Thewhilel oopallowstheusertocontinuetomakerequestsfordifferentdirect ions.Besurealltheremainingcodeisindentedwithinthewhileloo p.

whiletrue:

orig=input("Stringlocation:")

dest=input("Destination:")

url=main_api+urllib.parse.urlencode(\{ "key":key,"from":o rig,"to":dest $\})$

print("URL:"+(url))

json_data=requests.get(url).json()

json_status=json_data["info"]["statuscode"]

ifjson_status==0:

print("APIStatus:"+str(json_status)+"=Asuccessfulrouteca 11. $\ln ")$

JavaScriptObjectNotation(JSON)isaformatofscriptinglang uageforstoringandexchangingtextbetweenaserverandaclient.I npresentdaysJSONisbecomeverypopularformatthatwebbased servicesanddifferentAPIsareusingbecauseofitcaneasytoparse withmostmodernprogramminglanguagesincludingPython.

InJSONisobjectnotationhereobjectsareindicatedbycurlybr aces,assembledinPythondictionaries.JSONarraysareheldinsq uarebracketswhichareinPythonlists. Tosimplifythediscussion, wewillrefertotheJSONstructuresusingthefamiliarPythonterm s.Keepinmindhowever, thatJSONdataisusuallyconverted toPy thondatastructuresbeforeitisusedbyPythonprograms.

Published By: 


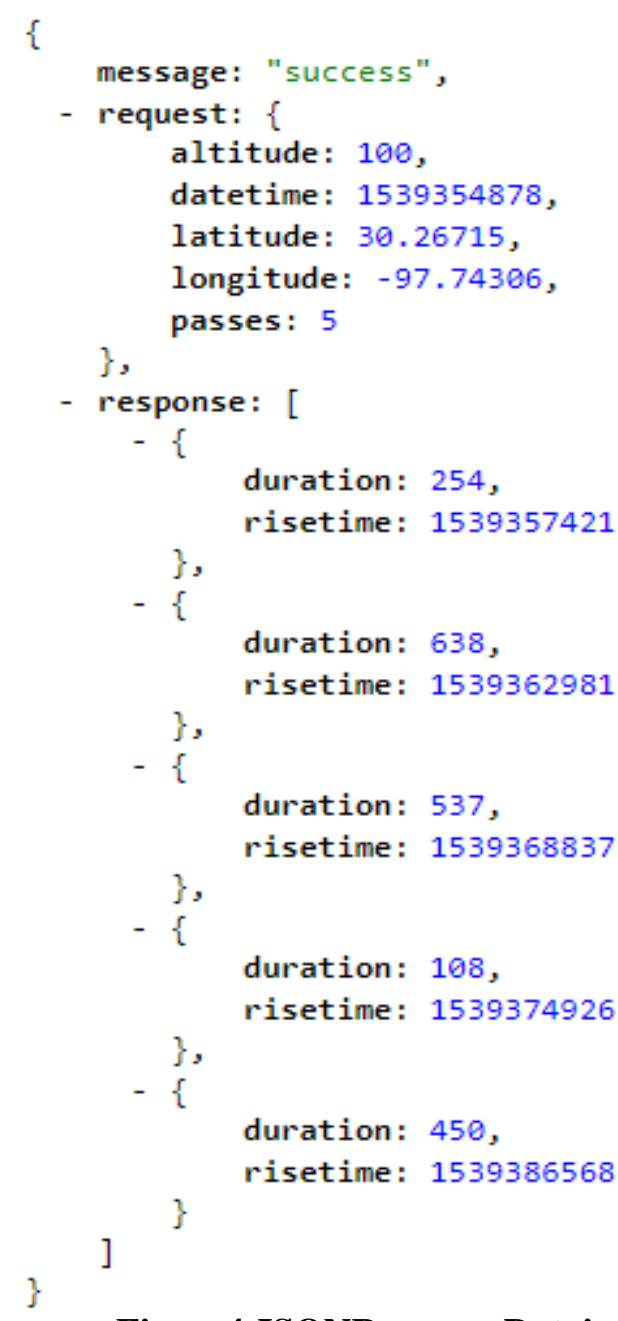

Figure4.JSONResponseDatainSD-WAN

IV. SD-

WANARCHITECTUREANDSERVICECOMPONE NTS

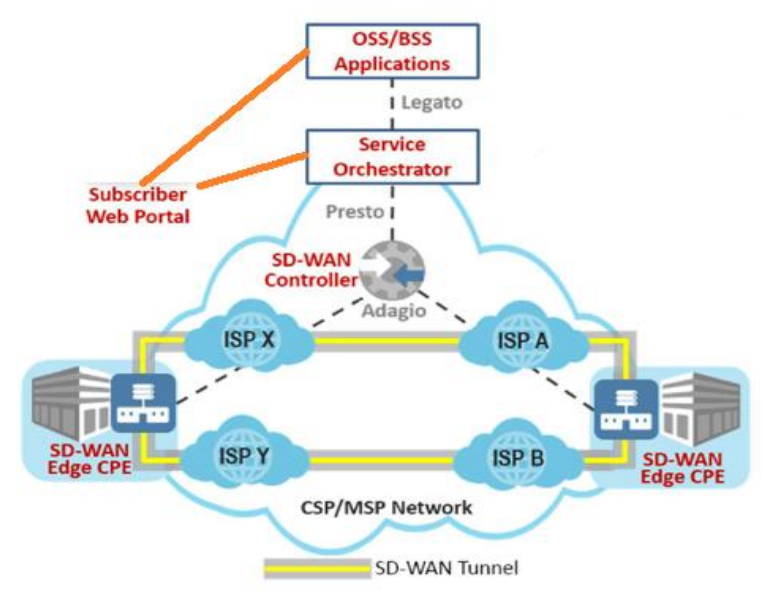

Figure5.SD-WANbasicArchitecture

TheSD-

WANarchitectureusetheservicecomponentstomanagetheserv icesinthenetwork.Thissectionwilldescribewhereeachoftheses ervicecomponents.

-SD-WANEdge

-SD-WANController

-ServiceOrchestrator

-SD-WANGateway

\section{- SubscriberWebPortal}

TheSD-

WANServiceComponentstounderstandtheirplacementinthear chitecture.ThedifferentSD-

WANservicecomponentsinacomprehensivenetworkdiagram. Tosimplifythediscussion, thispaperwillfocusonasingleprovide rdomainandthusnotcovertheinterfaceswhichareusedfortheco mmunicationofinter-providerservicefunctions [9][5].

\subsection{SD-WANEdge}

TheSD-

WANEdgecreatestheTunnelsforthevarioustypeswiredorwirel essunderlaynetworkslikeBroadbandInternet(DSL,CableandP ON),Wi-

FiandLTEwirelessnetworks, andIP(Internet)andMPLScorene tworks. ThisSD-

WANEdgeprovidestheservicedemarcationsameashoetheEthe rnetNIDprovidestheservicedemarcationforaCarrierEthernets ervice.ThefunctionalityoftheSD-

WANEdgeistocheckingapplication-

basedQoSandsecuritypolicyenforcement,applicationforwardi ngoveroneormoreWANconnections, andQoSperformanceme asurementsovereachWANtodetermineWANpathselection[10 ][11].TheSD-

WANEdgeoperatetheWANoptimizationfunctionssuchaspack etbuffering/reordering,datade-

duplication, datacompression, andforwarderrorcorrection.Sinc eSD-

WANEdgesoftenconnecttopublicInternetWANs,theywouldi nclude,ataminimum,someNATandfirewallcapabilities.

TheSD-

WANEdgeoperationsmaybeprovidedbyaphysicalCPEdevice residentontheclientpremisesandmanagedbytheCSPorMSP.S D-

WANEdgefunctionalitymayalsobeimplementedasasoftwarebasedvirtualnetworkfunction(VNF)whichmayrunonavirtual CPEattheclientpremisesoranyothertypeofgeneralizedcomput eplatform,e.g.,serverinadatacenter,whichmayalsobemanaged bytheCSPorMSPorbyacloudserviceprovider.TheMSPorCSP operatesandmaintainstheSD-WANEdgeaspartofanSDWANmanagedservice.

\subsection{SD-WANGateway}

TheSD-

WANGatewayinterconnectsthevariousclientssiteviaSDWANVPNnetworks.SD-

WANdeliveritsservicestoconnectedsitesintwoways.Onewayi sthroughtheSD-

WANEdgeconnectwiththesitespresentintheVPNnetwork[12] .AnotherwayisSD-

WANgatewayoperatestheconnectedsitesmeansinitiatetheserv icesandterminatingtheservices.SD-

WANservicefunctionalitiessuchasapplicationbasedtrafficforwardingovermultipleWANsorQoSandSecurit ypolicymanagementwillnotbeavailableattheMPLSVPNsitesb ecausetheydonothaveSD-

WANEdgeswhichperformthesefunctions[13]. 


\subsection{SD-WANController}

TheSD-

WANControllermanagesbothphysicalandvirtualdevicesforall SD-

WANEdgesandSDWANGatewaysassociatedwiththecontroll er.ThefunctionalityofSD-

WANcontrollerisnotonlyConfigurationandactivationofIPadd ressbutalsoitmaintainsconnectionstoallSD-

WANedgesandgatewaystoidentifytheoperationalstateofSDWANedgesacrossthenetworkandretrieve

betterQoSperformanceforeachSD-

WANedge[6][14].TheMSPorCSPoperatesandmaintainstheS D-WANControlleraspartandSD-WANmanagedservice.

\subsection{ServiceOrchestrator}

ThefunctionalityofServiceOrchestratorisprovidingtheservi cemanagementtotheSD-

WANservicelifecycleincludingfulfilmentofservice,performa nceofSD-

WAN,controlofarchitecture, assuranceforservices, usage,secu rityandpolicy[15].ThisoperatesandmaintainstheServiceOrche stratorwithanSD-

WANmanagedservice.TheServiceOrchestratoralsocanperfor mservicemodificationrequestsfromaSubscriberPortal.

\subsection{SubscriberWebPortal}

WhentheSD-

WANserviceisactivated, theSubscriberWebPortalconnectwit htheServiceOrchestratorformodifyingSD-

WANservicesuchassettingupdifferentQoS, securityorbusines spoliciesbasedonauser'srole,e.g., ‘view-

only'capabilityorabilitytomodifytheSD-

WANservice[7][16].TheServiceOrchestratoralsoperformserv icemodificationrequestsfromtheSubscriberPortal.

\section{CHALENGESTOIMPLEMENTSD-WAN\& RESULTS}

IndesigningofWideAreaNetworksvariouschallengesforthe designersespeciallyfortheSDNSystems. Thismayoccursduetol inkfailureandcomplexconnectivityinnetworkarchitecture.W ANnetworkarchitecturemainlyfocusonlinkproblems,complex ityofnetworksmorethanthedatacentriccontrol.Datacentersare dedicatedcontrolnetworkswithhighdegreeofparallellinksthes earedeployedinasafeandcontrolledenvironment.SDWANhad beenuseddistributedcontrolplane[17].ThemainchallengesofS DWANisupdatingthenetworkconfigurationinanetworkswitch inconsistentmanner, distributingtheSDNcontrollerstateandwh eretobeplacedthecontrollerinstances.

\subsection{DistributingSDNControllerState}

InmostoftheSDNdeploymentsfollowsacontrolprogramtode finetheoverallnetworkoperatingsystemwithcontrolanddatapla netogether.SDNcontrollerrepresentasinglecheckpoint,itisalso asinglepointoffailure[1][17].Toovercomescaleoutperformanc eandfaulttolerancedistributedSDNcontrollershadbeenpropos ed[7][11][16].SDNcontrollermainlyhashighthroughputrequir ements.SDNcontrollersinstancesdistributedinthenetworknatu
rallyalsorequiredistributingthecontrollerstate.Thestatesarege nerallymatchactionrules, topologyinformationandstatistics.O NOS(OpenNetworkOperatingSystem)isControllerarchitectur e.ONOSprovidestwomainfunctionalities:firstlyglobalabstract viewofthesharingnetwork.Secondly,extensivecapabilitiesfor bothperformanceandfaulttolerance.ONOSoperateseachswitc hasthecontrollerthatprogramsforwardingplane.Theswitchesdi stributedamongtallcontrollerinstances.

\subsection{PlacingControllerInstances}

IndeploymentofWideAreaNetwork(WAN)placingmultiple controllerscanleadsgreaterlatencyandfaulttolerance.Indeploy mentofcontrollersvariousquestionsmaybearisehowmanycont rollerstodeployandwheretoplacethem. Theresearcherswerered ucedtheplacementproblembasedonthedesiredmetric(1)whenu sesaverage-caselatencytheproblemreducedtominimumkmedianproblem.(2)whenworstcaselatencyitreducestothekcentreproblem.(3)whennodesnotexceedsaspecificlatencyitre ducestoNP-hardandboundtonextcontroller.

\subsection{UpdatingSDNSwitchesinaConsistentManner}

InSDWANnetworkarchitectureforwardingswitchesareuse dforenablingdynamicandcustomizeddataplaneconfigurations .Eachswitchpresentinthedataplanemustbeupdated.Updatingo fdataplaneinalargenetworkmaycausesevereinconsistencies.M ainreasonfortheseproblemsisinfeasibilitytoupdateentirenetw orksystemautomaticallytomainfullnetworkoperation. Theresu ltswitchesaregenerallyupdatedandconfiguredinstepbysteppro cedure.Inconsistencyinnetworkmayleadstointerruptedconnec tivity, forwardingloops, oraccessingcontrolviolations.Interme diateupdatestatesarecausingviolationinthebandwidth.Inordert oavoidthisproblemmostofthem,relyonplanninganexactupdate order.

\section{CONCLUSIONS}

WecanconcludetheSoftwaredefinedWideAreaNetworking overviewalongwithitsarchitecture.PresentdaySDWANbecom inghighlydemandingnetworkarchitecturebecauseofitsperfor mance,reliability, andimprovisedwideareanetworkissues.Inpa rticularWANweremoreexpensiveandneedshighperformanceh ardware.SDWANarchitectureoptimizestheWANnetworkand providesbetterscalabilityandfaulttolerance.SDWANtechnolo giesanddeploymentssustainsforlongertimeandcreatemoreimp actonthefutureoperationofWANS.SD-

WANimplementationwithCloudComputingisachallengingtas k.FromthispaperweexplainedaboutarchitecturalissuesintheS D-

WAN.Inthefeaturewecontinueourresearchwithoptimalpathtra cingbycontrolplaneandsecurityissuesintheSD-WAN.

\section{REFERENCES}

1. SmineshC.N,GraceMaryKanagaE,RanjithaK."AProactiveFlo wAdmissionandRe-

RoutingSchemeforLoadBalancingandMitigationOfCongestion PropagationInSDNDataPlane",InternationalJournalofCompute rNetworks\&Communications(IJCNC)Vol.10,No.6,November 2018,pp.(117-134). 
2. SallauddinMohmmad,DrM.Sheshikala,Shabana"SoftwareDefi nedSecurity(SDSec):Reliablecentralizedsecuritysystemtodece ntralizedapplicationsinSDNandtheirchallenges"JourofAdvRes earchinDynamical\&ControlSystems, Vol.10,10-

SpecialIssue,2018,pp.(147-152)

3. P.Berde,M.Gerola,J.Hart,Y.Higuchi,M.Kobayashi,T.Koide,B. Lantz,B.O'Connor,P.adoslavov,W.Snow, andG.Parulkar,ONO S:TowardsanOpen,DistributedSDNOS.InProc.oftheThirdWor kshoponHotTopicsinSoftwareDefinedNetworking,2014.

4. Risdianto,A.C.,Shin,J.andKim,J.,BuildingandOperatingDistrib utedSDN-

CloudTestbedwithyperconvergentSmartXBoxes,inProc.6thEA IInternationalConferenceonCloud

Computing,Daejeon,Korea,Oct.2015.

5. M.Caesar,D.Caldwell,N.Feamster,J.Rexford,A.Shaikh,andJ.va nderMerwe.DesignandImplementationofaRoutingControlPlatf orm.InProc.ofthe2ndConferenceonSymposiumonNetworkedS ystemsDesign\&Implementation.USENIXAssociation,2005.

6. Jain,S.,Kumar,A.,Mandal,S.,Ong,J.,Poutievski,L.,Singh,A.,Ve nkata,S.,Wanderer,J.,Zhou,J.,Zhu,M.andZolla,J.,2013,August. B4:Experiencewithaglobally-

deployedsoftwaredefinedWAN.InACMSIGCOMMComputer CommunicationReview(Vol.43,No.4,pp.3-14).ACM.

7. M.Casado,M.Freedman, andJ.Pettit.Ethane:Takingcontrolofthe enterprise.ACMSIGCOMMCCR,37(4),2007.

8. Berde,P.,Gerola,M.,Hart,J.,Higuchi,Y.,Kobayashi,M.,Koide,T .,Lantz,B.,O'Connor,B.,Radoslavov,P.,Snow,W.andParulkar,G .2014,August.ONOS:towardsanopen,distributedSDNOS.InPr oceedingsofthethirdworkshoponHottopicsinsoftwaredefinedne tworking(pp.1-6).ACM.

9. A.Greenberg,G.Hjalmtysson,D.A.Maltz,A.Myers,J.Rexford,G .Xie,H.Yan,J.Zhan, andH.Zhang.Acleanslate4Dapproachtonet workcontrolandmanagement.ACMSIGCOMMCCR,35,2005.

10. A.Gupta,R.MacDavid,R.Birkner,M.Canini,N.Feamster,J.Rexf ord,andL.Vanbever.AnIndustrial-

ScaleSoftwareDefinedInternetExchangePoint.In13thUSENIX SymposiumonNetworkedSystemsDesignandImplementation, mar2016.

11. Shah,SyedAbdullah,etal."AnarchitecturalevaluationofSDNcon trollers."Communications(ICC),2013IEEEInternationalConfer enceon.IEEE,2013.

12. A.Gupta,L.Vanbever,M.Shahbaz,S.P.Donovan,B.Schlinker,N. Feamster,J.Rexford,S.Shenker,R.Clark,andE.Katz-

Bassett.SDX:ASoftwareDefinedInternetExchange.InProc.ofth eACMSIGCOMM2014Conference, 2014.

13. Hock,David,etal."ParetooptimalresilientcontrollerplacementinSDNbasedcorenetworks."TeletrafficCongress(ITC),201325thIntern ational.IEEE, 2013.

14. D.Levin,A.Wundsam,B.Heller,N.Handigol,andA.Feldmann,“ Logicallycentralized?:statedistributiontradeoffsinsoftwaredefinednetworks,"inbHotSDN,2012.

15. B.Heller,R.Sherwood,andN.McKeown.TheControllerPlaceme ntProblem.InProc.ofthe1stWorkshoponHotTopicsinSoftwareD efinedNetworks, 2012

16. C.-

Y.Hong,S.Kandula,R.Mahajan,M.Zhang,V.Gill,M.Nanduri,an dR.Wattenhofer.AchievingHighUtilizationwithSoftwaredrivenWAN.InProc.oftheACMSIGCOMM2013Conference, 2 013.

17. D.Kothandaraman,C.Chellappan"EnergyEfficientNodeRankBasedRoutingAlgorithminMobileAd-

HocNetworks"InternationalJournalofComputerNetworks\&Co mmunications(IJCNC)Vol.11,No.1,pp45-61,January2019 